\title{
Démons, enfer et spectres chez Heym : entre sécularisation et fascination
}

Dämonen, Hölle und Geister bei Georg Heym, zwischen Säkulariserung und Faszination

Demons, hell, and ghosts by Georg Heym, between secularization and fascination

Dominique lehl

\section{(2) OpenEdition}

Journals

Édition électronique

URL : https://journals.openedition.org/ceg/10857

DOI : $10.4000 /$ ceg. 10857

ISSN : 2605-8359

Éditeur

Presses Universitaires de Provence

Édition imprimée

Date de publication : 1 octobre 2012

Pagination : 355-365

ISBN : 0751-4239

ISSN : 0751-4239

Référence électronique

Dominique lehl, « Démons, enfer et spectres chez Heym : entre sécularisation et fascination 》, Cahiers d'Études Germaniques [En ligne], 63 | 2012, mis en ligne le 07 septembre 2020, consulté le 04 juin 2021 URL : http://journals.openedition.org/ceg/10857 ; DOI : https://doi.org/10.4000/ceg.10857 


\title{
Démons, enfer et spectres chez Heym : entre sécularisation et fascination
}

\author{
Dominique IEHL \\ Université de Toulouse 2 - Le Mirail
}

Dans l'évolution des problèmes qui se rattachent à la poésie spectrale, l'œuvre de Georg Heym, composée au début du XX $\mathrm{X}^{\mathrm{e}}$ siècle, occupe une place paradoxale et singulière : paradoxale, car il est surprenant de voir un poète en apparence audacieux et moderne, co-fondateur de l'expressionnisme allemand, se consacrer avec une telle insistance à des motifs comme les démons, l'enfer, les fantômes, qui évoquent un temps révolu ; paradoxale, mais aussi singulière, car il réussit à prêter à cet univers fantomatique une force persuasive, à l'intégrer à sa vision du monde et à éclairer à travers lui des aspects essentiels d'une époque qui, en face du transcendant, hésite entre refus et fascination.

Heym, parmi les premiers expressionnistes, est considéré comme le poète de la ville au temps de la civilisation industrielle. La ville moderne est pour lui déjà le prélude de l'Apocalypse. Il en démasque le vide et l'inconsistance qui ont succédé à la splendeur vivante de la cité antique, à laquelle Heym est attaché par toutes ses fibres. La ville moderne n'incarne rien, sinon le déclin et la mort. Elle ne subsiste que pour être détruite, pour flamber dans les incendies que Baal, le Dieu de la ville, allume partout dans les rues. Cette image, centrale dans son œuvre, est encerclée de visions tantôt radieuses, quand il évoque la Grèce, tantôt sinistres quand il se tourne vers un présent dans lequel il étouffe et se consume (3. 164) ${ }^{1}$. Son œuvre se situe entre un rêve qu'il ne peut réaliser et une mort qui envahit peu à peu cette vie et la transforme en un univers fantomatique et macabre.

Ici apparaît, dans l'optique de la sécularisation, l'originalité de Heym. Car l'univers funèbre dans lequel il s'installe n'est pas un substitut littéraire, un regard curieux ou sceptique sur un monde étranger. Heym ne passe pas

1 Toutes les références à l'œuvre de Heym sont comprises dans le texte et empruntées à l'édition en 4 volumes : Georg HEYM, Dichtungen und Schriften, München, Ellerman, 1960, 1968. Les références indiquent le tome suivi de la page (t. 1 Poésie, t. 2 Proses et drames, t. 3 Journal, t. 6 Documents) 
comme Rimbaud une saison en enfer. Il est installé durablement, sinon définitivement, dans un enfer certes fort diffèrent de l'image qu'en ont livrée les diverses religions, mais qui, dans son étrangeté, sait s'imposer à l'imagination du lecteur.

Il convient, pour mieux comprendre la forme très originale de cet envahissement par le spectral, de s'interroger sur sa forme ultime, au terme de deux ans d'une création continue (1910-1911), qui s'achève sur une mort brutale.

Un des aspects majeurs de cette œuvre est un dualisme dont Heym est d'ailleurs parfaitement conscient. Il écrit en août 1909 :

J'aime tous ceux qui ont un cœur déchiré, j'aime Kleist, Grabbe, Hölderlin, Büchner. J'aime Rimbaud et Marlowe... J'aime tous ceux qui ne cessent de douter d'eux-mêmes, tout comme je doute de moi presque tous les jours $(3,129)$.

Mais il corrige ce portrait un an plus tard :

Ce en quoi je l'emporte sur Nietzsche, Kleist, Grabbe, Hölderlin, c'est que je suis plus, beaucoup plus vital qu'ils ne le sont, au bon comme au mauvais sens du mot $(3,138)$.

Heym est partagé entre un mal du siècle aigu, celui des années 1830 , de Grabbe et de Büchner, beaucoup plus virulent que le mal du siècle romantique, et un vitalisme bien connu de ses amis du Nouveau Club, comme John Wolfsohn, qui évoque "son rêve d'une élévation dionysienne du peuple tout entier au sein d'une grande nature pleine d'une vie animale, pénétrant l'homme et pénétrée par lui' $(6,146)$. Mais il est en même temps conscient de la fin de Dionysos qui cherche un refuge loin du dieu chrétien qui l'a exclu et chassé avec ses emblèmes sinistres $(1,14)$.

Ce dualisme se traduit dans les œuvres par la coexistence d'une poésie de la beauté et de la vie, et d'une poésie funèbre qui est très tôt le thème privilégié de Heym. Les premiers poèmes, entre 1903 et 1909, ont des titres sans équivoque : Le bateau des morts $(1,529)$, Le cimetière des pauvres $(1$, 524), Le vieux cimetière (1, 639), A ma tête de mort (1, 641), La maison morte $(1,559)$, Le squelette $(1,623)$, De profundis $(1,604)$, etc. Dans ces vers on trouve souvent, dans un mélange de douceur et d'amertume, une convention qui fait bientôt place à d'authentiques images de mort et de déclin. Une part de cette convention est conservée, sous une autre forme après 1910, dans des poèmes qui s'inspirent de la veine traditionnelle du romantisme funèbre. Ainsi la deuxième partie du Hollandais volant $(1,201)$, Visions noires $(1,212)$, La patrie des morts $(1,207)$, La Cène infernale $(1,231)$, les trois versions de La nuit (332.335). C'est une poésie des cryptes et des tombeaux qui unit un pittoresque douteux à des visions qui se veulent effrayantes mais qui restent peu convaincantes. Dans ces vers Heym est parfois proche du pittoresque fantomatique de la littérature anglaise du XIX siècle, et il s'inscrit dans un courant de sécularisation en transformant en paysage spectral une angoisse qui a perdu son caractère religieux. Mais ces 
poèmes ne sont qu'une faible part d'une œuvre poétique qui élabore une vertigineuse vision de la mort, et qui recrée un univers fantomatique original. Le décor devient alors réalité. En effet les visions spectrales de Heym sont d'abord la traduction d'une expérience personnelle, sous la forme d'une impuissance à donner à la vie une cohérence et un sens. Dans sa jeunesse il voue à Nietzsche un culte quasi religieux et semble fasciné par l'exemple admirable du surhomme. Mais trois ans plus tard, en 1909, son enthousiasme parait nettement atténué. Il écrit alors un bref essai : Ébauche d'une religion nouvelle $(2,164)$, dans un programme modéré et composite qui sauve des valeurs chrétiennes comme l'amour, mais en l'unissant à la force, qui conserve un Christ réduit aux dimensions humaines et qui place la Nature plus haut que l'Eglise dans une optique Renaissance. L'influence de Nietzsche est présente, mais tempérée. Il n'ouvre pas de débat sur le problème des rapports entre immanence et transcendance. Nietzsche dont, en 1907 encore, il s'exhortait à suivre l'exemple $(3,98)$, semble s'éloigner de lui. Mais cela ne signifie nullement un retour au christianisme. Dieu reste pour lui un épouvantail et ses rares évocations du Christ manquent beaucoup d'enthousiasme ${ }^{2}$. La seule évidence qui s'impose à lui est celle de l'inanité d'un monde définitivement corrompu. Ursula R. Mahlendorf se réfère à ce propos à un passage du Journal de juin-juillet 1910, qui succède à l'affirmation que la bonté de Dieu est impensable.

Beaucoup plus vraisemblable est l'hypothèse de la méchanceté de Dieu ou du destin. Car tout ce qui arrive dans le monde est le mal ou devient le mal $(3,136)$.

Elle en conclut que Heym est en proie à un pessimisme radical qui se traduit dans sa hantise des démons pernicieux et dangereux qui gouvernent le monde ${ }^{3}$. Il y a certes une part de vérité dans cette affirmation, car tout l'appareil fantomatique de Heym, démons, spectres, morts, enfer, est étroitement lié au tourment du poète, mais selon des modes d'expression très divers, qui prêtent à cet appareil une variété remarquable. Il existe toute une hiérarchie des démons et on ne saurait confondre Les démons avec Les démons des villes ou avec Le dieu de la ville.

A la tête des démons trône le dieu Baal qu'on peut tenir pour une version moderne du Baal légendaire connu pour la cruauté de ses sacrifices humains, ou tout simplement du Dieu terrible et vengeur de l'Ancien Testament. Ursula

2 L'image de Dieu apparaît sous forme d'une attaque violente dans les poèmes du début de 1910 : Dionysos (p. 14) ; Je te maudis, o dieu (p.18). La paresse et l'inertie de Dieu sont évoquées dans: Comme un oil regardant sans trouver de lumière (p. 21) et il ne retrouve une figure de père que dans l'énigmatique poème L'arbre (p. 490). Le Christ est présenté sous un jour très inquiétant dans La Cène infernale (p. 231) qui fait un amalgame de Satan et d'un christ chargé de l'amertume et de la rancœur d'un ange déchu. Dans Les fous, variation (p. 262), il n'est qu'une épave, plongée dans la détresse et l'abandon. Seul le poème Pilate (p. 486) montre le Christ dans sa splendeur, dans son envol vers le ciel.

3 Ursula R. MAHLENDORF, "The myth of evil. The reevaluation of the Judaic-christian tradition in the work of Georg Heym", in Germanic review, n 86, 1961, p. 192. 
R. Mahlendorf souligne fort justement une influence nette de la Bible et des textes chrétiens sur Heym, et découvre dans la figure de Baal la rencontre des civilisations grecque et juive, d'Arès et de Jéhovah ${ }^{4}$. Le poème est une vision d'apocalypse et peut évoquer le déclin de Babylone ou de Sodome et Gomorrhe. Mais on y découvre aussi d'autres aspects, à la fois surhumains et humains. Ce mélange est bien suggéré par les dessins à la plume de Heinrich Kley, connu de Heym, qui unissent un gigantisme terrifiant à des contorsions grotesques ${ }^{5}$. Kley trace la figure violente et brutale d'un incendiaire illuminé qui a bien sa place dans le monde moderne et illustre tout un processus de sécularisation : Karl Ludwig Schneider insiste à juste titre sur la complexité des démons de Heym ${ }^{6}$. Elle est très perceptible dans Les démons de la ville qui offrent toute une gamme de tons, entre l'horrible, l'effrayant et le grotesque, notamment dans la scène où les démons, pleins de curiosité, tendent le cou pour observer l'accouchement d'un enfant sans tête, et où ils ne dévoilent leur aspect mystérieux, gigantesque et dangereux que dans la dernière strophe.

Mais les démons deviennent soudain gigantesques.

La corne sur leur front déchire le ciel rouge.

Avec fracas la terre tremble au creux des villes

Autour de leur sabot enveloppé de flammes. $(1,187)$

La variété de ces figures apparaît plus nettement encore dans le poème Les démons, où les figures démoniaques ne sont plus désormais des envoyés de l'enfer mais des forces répandues dans la nature, tantôt sous la forme d'oiseaux effrayants cachés dans les branches, tantôt flottant en l'air, comme des danseurs, tantôt enfouis dans la vase des rivières, tantôt comme des figures noires, rondes et terrifiantes, hantant la nuit les chambres des mourants.

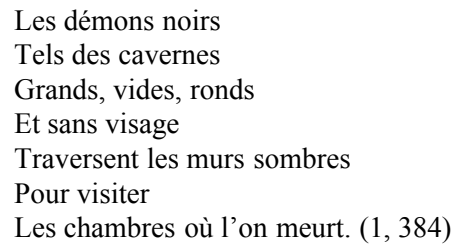

On découvre ici un trait essentiel chez Heym, une tendance à "démoniser" l'univers comme disent Karl Ludwig Schneider et Kurt Mautz, en le

4 Ursula R. MAHLENDORF, ibid., p. 182.

5 Voir à ce sujet Karl Ludwig SCHNEIDER, Georg Heym. Das lyrische Werk, München, DTV, 1977, 5. 611 .

6 SCHNEIDER insiste sur l'influence combinée de l'Apocalypse de Jean, de la mythologie antique, de Baudelaire et de Rimbaud.

7 Voir notamment Karl Ludwig SCHNEIDER, Der bildhafte Ausdruck in den Dichtungen Georg Heyms, Georg Trakls und Ernst Stadlers, Heidelberg, Winter, 1954, et Kurt MAUTZ, 
peuplant de figures indéchiffrables en marge de toute mythologie cohérente et qui se manifestent surtout comme un envahissement par des forces puissantes et étranges. C'est en ce sens que Heym peut évoquer dans un poème "le démon du feu solaire" $(1,249)$. Les démons relèvent ici à la fois du démoniaque et du démonique (au sens d'une puissance qui traverse l'univers). Le pessimisme radical de Heym est compensé par son dynamisme et son vitalisme. Dans cette imagerie, le rôle de l'animal est essentiel : chauvessouris et vampires $(3,11)$, lion fabuleux $(1,259)$, hérons fantomatiques $(1$, 338). Un rôle important revient aussi à la lune, qui perd chez Heym la poésie rêveuse et diaphane dont l'entourait le romantisme et qui devient une figure hargneuse et maligne "qui écrase la forêt" $(1,85)$, qui dresse, hystérique, pareille à une hydre, les longs cous qui portent ses têtes. Le passage du démoniaque au démonique correspond à une forme de sécularisation, en un sens non systématique.

A côté des démons, Heym décrit l'enfer et les enfers, dans une vision particulièrement intéressante, car la diversité qui marquait la peinture des démons fait place à une convergence significative dans l'interprétation du rôle et du sens du monde infernal, qu'il soit antique ou moderne. L'enfer chrétien est au centre d'un long poème en quatre parties L'enfer (327). Les deux premières parties nous entraînent dans un enfer banalisé. Le narrateur découvre à son arrivée un monde très semblable à la terre, monde de gens qui s'ignorent et s'ennuient et portent sur leur visage tous les stigmates des soucis quotidiens. Le diable qui apparaît dans la deuxième partie est un vieillard perclus de douleurs, affable et inoffensif, dépourvu en tout cas de tout caractère diabolique. Il évoque avec indulgence ses relations anciennes avec Dieu le père, se considère désormais comme "une fiction" et se sépare de ses visiteurs en leur offrant des cigares. Dans cette caricature du monde d'en bas, pratiquée fréquemment dans la littérature moderne, l'enfer est totalement sécularisé. Mais à la différence de divers contemporains qui le suppriment par la parodie, Heym a gardé dans la quatrième partie du poème une image réelle et tragique de l'enfer qu'il situe paradoxalement et ironiquement non pas au cœur du monde infernal, mais en marge, dans un lieu, les limbes, qui n'est que l'antichambre de l'enfer. Il modifie ainsi totalement la vision courante des limbes, car dans la théologie catholique les limbes sont un lieu de douceur et de félicité naturelle, malgré l'absence de Dieu. Certaines interprétations vont plus loin en permettant aux enfants non baptisés d'y accéder à la vie éternelle, dans la contemplation de Dieu. Les limbes sont dépourvus en tout cas de tout caractère tragique. Heym retourne la situation en décrivant les limbes comme le véritable enfer dans un paysage profondément sinistre ou les êtres sont suspendus dans un "vide aveugle" $(1,330)$, une "affreuse ankylose", "dans le labyrinthe immense de leurs tourments" $(1,331)$. Cet enfer se confond pour Heym avec la vie. Comme dans la vie, tout y est sous

Georg Heym. Mythologie und Gesellschaft im Expressionismus, Frankfurt a. M., Athenäum, 1972. 
le signe de l'effondrement, de la décomposition, dans une attente éternelle pire que la mort. Paradoxalement c'est dans le domaine inoffensif des limbes que Heym découvre le vrai visage de l'enfer, qui n'est pas pour lui, sauf dans les rêves de ses fous, un lieu de torture physique, mais de souffrance de l'âme, dans l'affreuse ankylose d'une époque livrée à l'immobilité et à l'inertie.

La convergence dans le poème d'un enfer inoffensif et d'une effrayante antichambre de l'enfer (qui est en réalité la vraie forme de l'enfer), montre bien toute la complexité des rapports de Heym avec les motifs de l'univers infernal. Il les banalise d'abord pour leur redonner à la fin une intensité extrême qui les situe en dehors de toute perspective de réduction. Par ailleurs il retrouve une forme de sécularisation en assimilant, sous le signe de l'écrasement et de l'asphyxie, l'enfer et la vie.

Sa conception de l'enfer est très cohérente, qu'il s'agisse du monde chrétien ou du monde antique. Ce que les morts cherchent tous dans l'Hadès, c'est d'abord une libération et ensuite un lieu d'accueil qui leur offre une compensation au vide effrayant de la vie. C'est pourquoi ils attendent patiemment, dans La morgue $(1,286)$ et d'autres poèmes, l'arrivée du guide qui les mènera - croient-ils - à bon port. Mais ils connaissent une double déception : celle de rester sur terre, ou de ne découvrir dans le monde souterrain qu'un lieu d'isolement, de confusion et de tristesse qui correspond à la déréliction que décrit le poème Les limbes.

Des chambres, des couloirs, des niches, des lieux sombres

Pareils aux niches installées dans l'arbre creux,

Dans l'empire d'Hadès sont dispersées dans l'ombre,

La vague les conduit par ces sombres refuges.

(Marathon. 1, 38)

La mort réserve aux morts toujours le même accueil.

\footnotetext{
Et les morts cependant vivent au fond des tombes,

Dans de petites chambres pour l'éternité.

O cet immense empire aux innombrables salles.

Aux pièces ressemblant aux noirs terriers des taupes,

Avec mille couloirs où se croisent les morts

Furtifs et tristes, sans jamais se reconnaître.

(Mors. 1, 132)
}

La mort conserve l'anonymat, l'angoisse et le vide de la vie, et Heym ne se fait aucune illusion. Parmi les morts qui espèrent, seuls les fous sont convaincus qu'ils redeviendront des arbres et retrouveront ainsi, par une réincarnation dans la nature, l'existence dionysienne qui sur terre s'est révélée impossible. Mais à la fin de Morgue ne subsiste que l'attente du néant : "dans le néant serons-nous miettes ?" $(1,290)$.

Il n'en reste pas moins que Heym est toujours fasciné par la recherche d'un havre dans la mort et par une réincarnation dont il ne peut déterminer la forme, ni le lieu, que ce soit, comme le suggère le poème Courage! $(1,365)$, 
dans la Babylone des morts aux mille rues, dans un vol nocturne au-dessus des forêts, au milieu des éclairs ou dans le calme des champs. Tout lui échappe et se dissipe, malgré son profond besoin de retrouver dans la mort l'existence dionysienne qu'il n'a jamais pu réaliser sur terre.

Ici apparaît un trait majeur de l'univers de Heym qui, à bien des égards, est plus spectral qu'infernal et où les fantômes l'emportent sur les démons. La "démonisation" chez Heym est pour une bonne part l'expression de sa fantaisie et de son vitalisme, qui peuplent l'univers de forces puissantes et souvent incohérentes qui animent le feu de l'imagination d'un poète expressionniste doublé d'un visuel, qui sait concilier le mystère des ailes et l'étrangeté des becs et des serres.

La présence constante des spectres dans son cuvre se situe à un autre niveau et traduit son attirance profonde pour la mort. Cette "fantomisation" revêt des formes diverses et devient de plus en plus marquée au cours de l'année 1911. A côté de Baal, des démons des villes et de la nature, un rôle essentiel revient aux figures de l'errance et de l'oubli, et plus encore aux morts qui sont avec les vivants dans une relation d'impuissance et d'attirance. Les morts sont répandus partout, sous les pieds des vivants qu'ils implorent et qui ignorent leur appel. Ainsi les fantômes des suicidés dont "personne n'écoutait le langage" (Les suicidés. 1, 473) et qui restent prisonniers entre le sommeil et le rêve. Cette situation est nettement suggérée dans la figure du Hollandais volant éternellement fixé à la barre de son navire.

Le masque d'une éternité morte

A recouvert ses traits de la glace du vide $(1,195)$.

Temps figé, temps glacé, éternité vide, tels sont les traits majeurs de l'univers spectral de Heym. Il s'agit ici d'une paralysie et d'une extinction de la vie qui perd toute couleur et devient systématiquement morne et vide, ainsi dans le long poème Tétralogie d'automne qui s'achève sur une strophe significative.

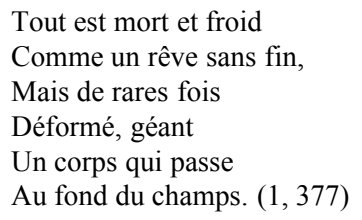

Le poème décrit un engourdissement d'abord anodin, qui gagne peu à peu tous les êtres qui s'estompent et s'effacent, ne laissant plus subsister qu'une ombre. De plus en plus Heym est attiré par le creux et le vide et une nuit peuplée de fantômes inquiétants. L'image des villes se fantomatise. Elles sont décrites dans leur effondrement. Villes "creuses comme des tombes et tombant en ruines" (Voici soudain surgir la grande mort. 1, 422). Villes "vides et oubliées" (Et les cors de l'été se sont tus. 1, 321). Dans ces villes fantômes errent d'étranges figures: "l'amas des villes mortes où errent les 
meurtriers" (Les villes. 1, 494). Ailleurs les meurtriers sont des fantômes à l'affût, dans un monde de mourants. Ainsi dans la troisième partie de Tétralogie d'automne.

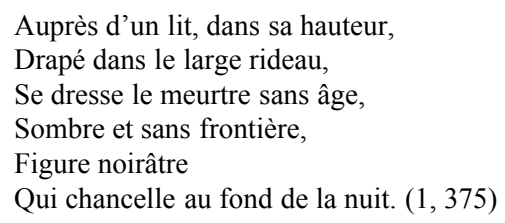

A ce stade de sa création, Heym plonge tout le réel dans une étrangeté fantomatique qui marque le monde moderne comme les ruines du monde ancien. Les nouvelles maisons qu'il décrit $(1,390)$, ne sont pas seulement la critique d'une architecture inepte, mais surtout une image de l'irréalité de la ville moderne où les maisons n'offrent aucun refuge contre le froid ni contre les voleurs, figures fantastiques qui rôdent avec leurs lanternes sur les toits et dans les escaliers. A ce niveau le fantomatique se transforme en perception du vide, dans un décor très différent de celui des tombeaux et des cryptes dans certains poèmes de 1910. Il s'agit maintenant d'un décor d'extinction, qui correspond au vertige de Heym prisonnier d'un monde où il étouffe, comme il décrit directement la fin du monde sans explosions ni cataclysmes mais par simple épuisement.

Ces dernières images permettent de situer mieux Heym par rapport au problème de la sécularisation du religieux. Rien ne portait l'auteur de Marathon vers une poésie de la fin et du néant, sinon l'échec de sa tentative dionysienne qui le ramène à l'autre composante de sa nature, la dépression et la mort. C'est le mystère de la mort qui fait le tragique de la poésie de Heym et qui permet de préciser sa place dans le courant de sécularisation. Mais il faut se garder de lui appliquer des critères définitifs. Une partie de son œuvre décrit des fantômes et des démons quasi traditionnels. Mais très tôt il transforme les démons en figures vivantes autant qu'étranges dans lesquelles on retrouve la marque de l'incohérence universelle. A ce stade, l'imagination de Heym fonctionne de concert avec sa volonté de secouer la torpeur du monde, de la peupler de forces qui retrouveraient l'héritage du grand élan dionysien perdu. Mais la démonisation se révèle n'être qu'un substitut fictif de cet élan, et elle se transforme finalement en une fantomisation qui dure jusqu'à la mort du poète. Sa création devient alors l'expression d'une aliénation profonde ${ }^{8}$. Les fantômes traditionnels rattachaient les vivants aux morts par les regrets, le remords, les souvenirs et le désir. Dans les derniers poèmes la fantomisation est universelle. Elle ne confronte pas deux mondes mais confond tout dans la même irréalité et la seule expérience réelle est celle du vide. Dans l'univers obscur de Heym, l'ombre a une place essentielle. Chez Trakl on découvre aussi la présence de l'ombre mais Trakl conserve

8 Voir à ce sujet Herman KoRTE, Georg Heym, Stuttgart, Metzler, 1982. 
toujours en lui l'aiguillon du mal qui le ramène, dans le souvenir de l'inceste, à son implacable et ineffaçable expérience personnelle. On aperçoit ici ce qui le sépare de Heym pour qui le mal a un sens différent. En dépit des apparences, Heym n'est pas véritablement un poète du mal. Ursula R. Mahlendorf voit en lui le créateur d'un mythe du mal ${ }^{9}$ qu'elle découvre dans sa croyance définitive en l'échec des entreprises humaines et dans sa conviction, déjà notée, que Dieu ne peut être qu'un être méchant qui rejette $1^{\prime}$ 'homme ${ }^{10}$. En fait il faut préciser cette image à travers celle, qui revient souvent chez Heym, d'un Dieu fainéant, parasite, bon à rien. Le mal, chez Dieu, c'est la paresse, l'inertie, c'est le refus d'améliorer le monde : "tu restes immobile" $(1,22)$. A la lumière de ces affirmations on comprend mieux ce qu'est le mal pour Heym qui ignore les convictions morales et ne rêve que de catastrophes qui mettraient fin à la torpeur qui a envahi le monde moderne et qui rend impossible le retour à la grande harmonie dionysienne. Il ne découvre à la fin qu'un vide universel d'où le mal et le bien sont également exclus, et où tout se confond dans une commune paralysie. On est loin ici des catégories morales inséparables de toute compréhension du mal. Faut-il parler d'une forme ultime de sécularisation parvenue à un sommet où sont réduits à néant tous les domaines où elle pourrait s'appliquer, ne laissant plus persister qu'un souffle moribond d'esthétisme?

En fait Heym évite cette impasse par deux échappées ultimes de sa poésie. Il évite l'écueil de l'esthétisme systématique par les rapports ambigus qu'il entretient toujours avec l'image du monde. La tentation du vide qui marque le regard qu'il pose sur le désert de la terre et des eaux s'accompagne d'un vertige positif devant l'immensité mystérieuse et l'infinie tragédie des cieux ensanglantés pour lesquels il éprouve une admiration proche de la fascination. Dans cette ferveur constante, le religieux et l'esthétique sont unis en un mélange qui n'a rien d'artificiel.

Par ailleurs il échappe à la séduction de la mort et du néant par une solution originale. Parmi les figures fantomatiques qui hantent ses derniers poèmes, un rôle essentiel revient aux déshérités et particulièrement aux fous : fasciné par le phénomène de l'enfermement et de l'isolement, Heym a composé plusieurs poèmes sur les malades, les prisonniers, les aveugles, les sourds et surtout sur les fous et leur mystère. Il n'est pas le seul dans son époque à s'intéresser à eux, mais son originalité est liée à l'interprétation nouvelle qu'il en donne. Ces exclus ont en effet une grandeur et une présence qui fait d'eux les seuls êtres réels dans un monde qui expire. Loin de voir en eux, comme les naturalistes, de simples victimes de la misère, il explore précédé par le Rilke de Malte Laurids Brigge - la structure mystérieuse qui leur confère une place exceptionnelle parmi des vivants aveugles qui ne voient pas qu'ils sont déjà morts. On ne saurait parler ici de sécularisation,

9 Ursula R. MAHLENDORF, “The myth of evil”, p. 198

10 Ibid., p. 192. 
mais d'un renouveau subtil du sentiment religieux à travers une empathie qui se transforme en une sorte d'envoûtement.

Cette évolution permet de mieux comprendre toutes les contradictions de Heym et ses difficultés dans la lutte qu'il entreprend pour s'en libérer. Il est par là beaucoup plus proche de son temps qu'il n'y paraît. Le drame de Heym est de n'avoir pas trouvé, après son détachement du monde familial et de la morale chrétienne, un autre univers où il aurait pu se libérer de son angoisse.

Ses relations avec Nietzsche sont à cet égard très révélatrices et éclairent un aspect essentiel de l'époque. Ce que le jeune homme admire en Nietzsche, c'est moins l'abolition de la morale que sa sublimation. Le surhomme est pour lui une forme d'élévation, un modèle idéal. " $\mathrm{O}$, si je pouvais réussir à transformer ma vie, pour devenir une flèche volant vers le surhomme" (3, 44. mars 1906). La suite de son Journal évoque peu Nietzsche, sauf pour constater qu'il n'a pas été encore capable de suivre son exemple $(3,98$. octobre 1907). Son essai de 1909 sur une religion nouvelle est le reflet d'un nietzschéisme tempéré. Certes il demeure proche de Nietzsche dans son rejet du christianisme, mais toute son adoration pour la Grèce reste toujours sur le plan de la nostalgie, et son dionysisme se réduit à un rêve. La mythologie grecque ne lui est pas d'un grand secours, sinon dans l'enchantement qu'il puise dans quelques très beaux paysages. Il garde de l'enfer grec la vue désolante d'une prison anonyme, et l'enfer chrétien reste, dans l'image des limbes, très proche des enfers, dans une commune angoisse, et dans la même attente douloureuse et vaine d'une révélation dans la mort.

Une comparaison entre Heym et Nietzsche montre, à côté de nombreuses affinités, des différences essentielles qui illustrent les contradictions de l'époque. Le philosophe Nietzsche détruit d'abord, par une analyse rigoureuse et subtile, tout un univers de mensonge. Mais il jette en même temps les bases d'une construction beaucoup plus incertaine, qui relève de la poésie autant que de la philosophie, et dans laquelle l'univers immanent acquiert un pouvoir et une portée illimités qui ont séduit, à travers l'image de l'homme nouveau, une génération fascinée par l'utopie.

Heym, de son côté, troque une poésie de la louange nostalgique et de la vision agressive contre une poésie du vertige qui répond aux attentes d'une époque hantée par l'apocalypse. Mais, dans ce cheminement il passe du style de l'admiration et de la "démonisation" à une forme de "fantomisation" qui marque ses derniers poèmes. Le réaliste Heym, qui se dit "poète des choses" $(3,164)$ reste prisonnier d'un sentiment d'aliénation qu'il étend au monde entier.

On est ici bien loin de Nietzsche. On ne pourrait les rapprocher qu'en tenant la surhumanité de Nietzsche pour l'expression d'une morale de l'immanence réduite à elle-même, satisfaite d'elle-même et dépouillée de tous les leurres du transcendant. On peut songer à ce propos à l'immanence heureuse du rêve dionysien de Heym, mais la théorie nietzschéenne de l'amor fati évoque un mélange d'épicurisme et de stoïcisme étranger au réalisme vitaliste aussi bien qu'au déchirement de Georg Heym. La solution qu'il 
découvre pour se libérer de l'inertie et du déchirement est originale. Il y parvient grâce au recours à ce qu'on pourrait appeler une transcendance négative. Nietzsche se hisse vers le surhomme, Heym s'abaisse vers les "sous-hommes". Il n'est pas l'apôtre d'un humanisme qui exalte à tout prix la vie malgré tous les dénis qu'elle lui apporte, mais l'explorateur d'une humanité infime, située tragiquement en marge de l'humain, et dont le fascinant mystère lui indique, dans sa négativité même, un chemin vers un sens et une issue, vers cette "petite porte" évoquée dans le poème Le singe (1, 199).

Ici apparaissent la diversité et les possibilités d'un poète que l'on pourrait croire enfermé dans une forme d'immobilité. Heym n'a cessé de lutter contre les forces négatives qui paralysent l'homme et qu'il incarne dans des images mobiles de l'enfer, du diable et des démons qu'il sécularise tour à tour, dans leur forme antique et moderne, mais avec une variété parfois non dépourvue d'humour, ainsi dans le portrait du diable et parfois des démons (cf. Les Démons de la ville).

Paradoxalement c'est son besoin d'action, son vitalisme insatisfait qui l'amènent à un pessimisme radical auquel il échappe seulement dans une rencontre avec le mystère du ciel et sa plongée dans l'univers fascinant des déshérités.

C'est encore le mystère qui accompagne l'obstination des morts à trouver un havre en enfer. La réflexion sur le monde spectral n'est jamais un jeu, mais traduit son inquiétude profonde devant le sens de la mort et le destin des morts. Doté d'une lucidité dangereuse, Heym a su se préserver d'une relativisation extrême en restant fasciné par la beauté tragique du monde, la terre immense et désertique, le drame des cieux sans limites, l'éternité vide, autant que par les rigueurs de l'hiver pétrifié et le vertige de déréliction qui accompagne l'automne. Il garde toujours intacte une admiration qui se complète par un sens aigu du mystère des choses et des êtres. Ce sont là dans son œuvre des points fondamentaux de résistance, grâce auxquels il évite l'écueil d'une sécularisation réductive et simplifiante. Cela lui confère une place exceptionnelle parmi les artistes qui avaient, au début du $\mathrm{XX}^{\mathrm{e}}$ siècle, entrepris la tâche difficile de renouveler en profondeur la vision de leurs devanciers. 